\title{
26403 - ECHOCARDIOGRAPHY IS INSTRUMENTAL IN SECOND-ORDER CHORDAL CUTTING TO RELIEVE MITRAL LEAFLET TETHERING AND REDUCE MITRAL REGURGITATION IN PATIENTS WITH ISCHEMIC MITRAL REGURGITATION
}

\section{Asim Alam BSc, Michael Borger, MD PhD; Murphy Murphy, University Of Toronto, Toronto, ONTARIO, Canada}

Introduction: Chronic ischemic mitral regurgitation (IMR) is associated with apical displacement of papillary muscles leading to tethering of second-order chords and decreased leaflet coaptation [1]. Surgical division of second-order chords has been proposed as a novel method to counteract this mechanism [2]. Transesophageal and transthoracic echocardiography is instrumental for the diagnosis and surgical management of IMR. This study elucidates clinical and echocardiographic data which evaluate the efficacy of chordal-cutting in surgical management. Methods: We compared echocardiographic data in two groups of patients undergoing mitral valve repair, patients undergoing chordal-cutting procedure and those undergoing conventional mitral repair. TEE/TTE was utilized to measure tent height (the distance from the apex of the anterior mitral valve leaflet (AMVL) to the mitral annulus), tent area (the area of mitral leaflet extension into the left ventricle during systole) and distance between the AMVL apex and posterior left ventricular (LV) wall in IMR patients who underwent either chordal-cutting MV repair ( $n=15$ ) or conventional MV repair (control, $n=26)$ both preoperatively and postoperatively. Mitral regurgitation and LV ejection fraction (LVEF) were also measured. Results: Chordal-cutting patients were more likely to have an LVEF of less than $40 \%$ (100 vs. 41\%, p<0.001), higher prevalence of peripheral vascular disease ( $40 \%$ vs. $7 \%, \mathrm{p}=0.01)$, diabetes mellitus type II (53\% vs. $22 \%, \mathrm{p}=0.04)$, older age (69 \pm vs. $63 \pm$ years, $\mathrm{p}=0.05)$ and require urgent surgical management $(67 \%$ vs. $37 \%, \mathrm{p}=0.05$ ) than control patients. Complication rates were similar for the two groups, perioperative mortality occurred with a solitary chordal-cutting patient $(7 \%$ vs. $0 \%$ for control). Echocardiographic predictors of successful surgical management of chronic IMR were evaluated between the two groups. The chordal-cutting patients had greater reductions in tent area and in the distance between the AMVL apex and posterior LV wall ( $30 \%$ vs. $12 \%, \mathrm{p}=0.003)$. Chordal-cutting patients had lower recurrence of MR in the early postoperative period in comparison to control patients ( $0 \%$ vs. $19 \%, \mathrm{p}=0.05)$. Furthermore, chordal-cutting did not adversely affect postoperative LVEF (3\% mean reduction in chordal-cutting patients vs. $1 \%$ in the control group, $\mathrm{p}=0.9$ ). Discussion: Chordal-cutting decreases MV leaflet tethering and reduces early MR recurrence in patients with chronic IMR, without compromising LV function. Novel TEE/TTE measurements are instrumental in this procedure and should be familiarized by relevant cardiac anesthesiology professionals.

References:

1. Circulation 1997;96:1999-2008.

2. Circulation 2003;108:II111-II115. 